\title{
Motion of four-dimensional rigid body around a fixed point: an elementary approach. I.
}

\author{
A.M. Perelomov * \\ Departamento de Física Teórica, Universidad de Zaragoza, \\ E-50009 Zaragoza, Spain
}

\begin{abstract}
The goal of this note is to give the explicit solution of Euler-Frahm equations for the Manakov four-dimensional case by elementary means. For this, we use some results from the original papers by Schottky [Sch 1891], Kötter [Koe 1892], Weber [We 1878], and Caspary [Ca 1893]. We hope that such approach will be useful for the solution of the problem of $n$-dimensional top.
\end{abstract}

1. The equations of motion for a rigid body in a four-dimensional Euclidean space with a fixed point coinciding with the center of mass (and also for the $n$-dimensional case) are the generalization of famous Euler's equations. They were found first by Frahm [Fr 1874] ${ }^{1}$ and they have the form

$$
\dot{l}_{i j}=\sum_{k=1}^{4}\left(l_{i k} \omega_{k j}-\omega_{i k} l_{k j}\right), \quad \omega_{i j}=c_{i j} l_{i j}, \quad l_{i j}=-l_{j i}, \quad i, j=1, \ldots, 4 .
$$

Here $c_{i j}=I_{i j}^{-1}$, the dot denotes the derivative with respect to time $t$, and $l_{i k}, \omega_{j k}$, and $I_{i k}$ are components of angular momentum, angular velocity and principal momenta of inertia tensors, respectively.

\footnotetext{
* On leave of absence from Institute for Theoretical and Experimental Physics, 117259 Moscow, Russia. Current e-mail address: perelomo@dftuz.unizar.es

${ }^{1}$ The problem of generalization of Euler's equations was posed by Cayley [Ca 1846].
} 
In this paper we consider completely integrable Manakov's case [Ma 1977], when quantities $c_{i j}$ have the form ${ }^{2}$

$$
c_{i j}=\frac{b_{i}-b_{j}}{a_{i}-a_{j}} .
$$

In a number of papers (see [AM 1982], [Ha 1983], [AM 1988], and references therein) so called method of linearization on the Jacobian of a spectral curve defined by the characteristic polynomial of one of the matrix in the Lax pair was used. However, as it was mention in [AM 1988], "this approach has remained unsatisfactory; indeed (i) finding such families of Lax pairs often requires just as much ingenuity and luck as to actually solve the problem; (ii) it often conceals the actual geometry of the problem".

So, in the present note we return to the original Schottky-Kötter approach [Sch 1891], [Koe 1892]. In our opinion, this elementary and natural approach is more adequate for the problem under consideration. We hope that it will be useful also for the more complicated problem of $n$-dimensional top at $n>4$.

Let us remind that in the paper [Sch 1891] the problem under consideration was reduced to the Clebsch problem [Cl 1871] of the motion of a rigid body in an ideal fluid ${ }^{3}$. For the special cases, the last problem was integrated explicitly by Weber [We 1878] and by Kötter [Koe 1892].

However, the Clebsch problem is related not to so(4) Lie algebra but to the $e(3)$ Lie algebra - the Lie algebra of motion of the three-dimensional Euclidean space. Hence, it is important to extend the Schottky-Kötter approach to give the solution in so(4) covariant form. Here we give such a solution using the elementary means ${ }^{4}$.

2. Note first at all that equations (1) are Hamiltonian with respect to the Poisson structure for the $s o(4)$ Lie algebra - the Lie algebra of rotations of the four-dimensional Euclidean space,

$$
\left\{l_{i j}, l_{k m}\right\}=l_{i m} \delta_{j k}-l_{i k} \delta_{j m}+l_{j k} \delta_{i m}-l_{j m} \delta_{i k} .
$$

\footnotetext{
${ }^{2}$ Note that for the "physical" rigid body $c_{i j}=I_{i j}^{-1}, I_{i j}=I_{i}+I_{j}$. In this paper we consider a general integrable case when quantities $c_{i j}$ and $I_{i j}$ are arbitrary.

${ }^{3}$ This result was rediscovered one century later in the paper [Bo 1986].

${ }^{4} \mathrm{~A}$ special $s o(4)$ case with tensor $l_{j k}$ of rank 2 was integrated explicitly by Moser [Mo 1980].
} 
The Hamiltonian is given by the formula

$$
H=\frac{1}{2} \sum_{j<k}^{4} c_{j k} l_{j k}^{2}
$$

where quantities $c_{i j}$ are given by formula (2), and equations (1) may be written in the form

$$
i_{j k}=\left\{H, l_{j k}\right\} \text {. }
$$

Let us remind that equations (1) have four integrals of motion

$$
\begin{gathered}
H_{0}=l_{12} l_{34}+l_{23} l_{14}+l_{31} l_{24}=h_{0}, \\
H_{1}=\sum_{j<k}^{4} l_{j k}^{2}=h_{1}, H_{2}=\sum_{j<k}^{4}\left(a_{j}+a_{k}\right) l_{j k}^{2}=h_{2}, H_{3}=\sum_{j<k}^{4} a_{j} a_{k} l_{j k}^{2}=h_{3} .
\end{gathered}
$$

Note that $H_{0}$ and $H_{1}$ are the Casimir functions of $s o(4)$-Poisson structure, and the manifold $\mathcal{M}_{h}$ defined by equations (6) - (7) is an affine part of twodimensional Abelian manifold (see Appendix by Mumford to the paper [AM 1982]) ${ }^{5}$. Then formula (5) defines Hamiltonian vector field on $\mathcal{M}_{h}$.

The main result of this note is the following one: by elementary means, it is shown that the dynamical variables $l_{j k}(t)$ are expressed in terms of Abelian functions $f_{j 4}\left(u_{1}, u_{2}\right), f_{k l}\left(u_{1}, u_{2}\right), f_{0}\left(u_{1}, u_{2}\right)$, and $g\left(u_{1}, u_{2}\right)$ related to genus two algebraic curve

$$
y^{2}=\prod_{j=0}^{4}\left(x-d_{j}\right), \quad d_{0}=0, \quad d_{4}=d_{1} d_{2} d_{3},
$$

with arguments depending linearly on time.

Theorem. Solution of equations (1) has the form

$$
\begin{aligned}
m_{j} & =l_{k l}=g\left(u_{1}, u_{2}\right)\left(\alpha_{j} f_{k l}\left(u_{1}, u_{2}\right)+\beta_{j} f_{j 4}\left(u_{1}, u_{2}\right)\right), \\
n_{j} & =l_{j 4}=g\left(u_{1}, u_{2}\right)\left(\gamma_{j} f_{k l}\left(u_{1}, u_{2}\right)+\delta_{j} f_{j 4}\left(u_{1}, u_{2}\right)\right) .
\end{aligned}
$$

Here $(j, k, l)$ is a cyclic permutation of $(1,2,3), \alpha_{j}, \beta_{j}, \gamma_{j}, \delta_{j}$, and $d_{j}$ are algebraic functions of integrals of motion and quantities $a_{j}$ and $b_{k}$. Explicit expressions for them are given by (24)-(26), (34), (35), (41), and (44).

\footnotetext{
${ }^{5}$ I am grateful to A. N. Tyurin for the explanation of algebraic geometry related to this Appendix.
} 
Proof. The key problem is the "uniformization" of the manifold $\mathcal{M}_{h}$, i.e., finding of the "good" coordinates on it. The proof consists of several steps.

A. Following Kötter [Koe 1892] and using the linear change of variables $m_{j}$ and $n_{j}$ to new variables $\xi_{j}$ and $\eta_{j}$, we transform equation (7) to the more appropriate form:

$$
\sum_{j=1}^{3}\left(\xi_{j}^{2}+\eta_{j}^{2}\right)=0, \quad \sum_{j=1}^{3} \xi_{j} \eta_{j}=0, \quad \sum_{j=1}^{3}\left(d_{j} \xi_{j}^{2}+d_{j}^{-1} \eta_{j}^{2}\right)=0 .
$$

For this, following Schottky [Sch 1891], let us introduce the three-dimensional vector $\mathbf{l}(s)$ depending on parameter $s$ :

$$
\mathbf{l}(s)=\left(l_{1}(s), l_{2}(s), l_{3}(s)\right), \quad l_{j}(s)=\sqrt{s_{j 4}} m_{j}+\sqrt{s_{k l}} n_{j},
$$

where

$$
m_{j}=l_{k l}, \quad n_{j}=l_{j 4}, \quad s_{j k}=\left(s-a_{j}\right)\left(s-a_{k}\right),
$$

and $\{j, k, l\}$ is a cyclic permutation of $\{1,2,3\}$. It is easy to check that the function

$$
f(s)=\mathbf{l}(s)^{2}=\sum_{j=1}^{3} l_{j}(s) l_{j}(s)
$$

does not depend on time. So, it is the generating function of integrals of motion

$$
f(s)=h_{1} s^{2}-h_{2} s+h_{3}+2 h_{0} \sqrt{G(s)}, \quad G(s)=\prod_{j=1}^{4}\left(s-a_{j}\right) .
$$

From formulae (12) and (14) it is easy to get the Lax representation ${ }^{6}$

$$
\dot{L}(s)=[L(s), M(s)]
$$

where $L(s)$ and $M(s)$ are antisymmetric matrices of the third order corresponding to vectors $\mathbf{l}(s)$ and $\mathbf{m}(s)$,

$$
\mathbf{m}(s)=\left(m_{1}(s), m_{2}(s), m_{3}(s)\right), \quad m_{j}(s)=\sqrt{s_{k l}} m_{j}+\sqrt{s_{j 4}} n_{j},
$$

\footnotetext{
${ }^{6}$ However, this representation does not need for the proof of Theorem. For the generalization of such representation for the $n$-dimensional case see [Fe 2000].
} 


$$
L(s)=\left(\begin{array}{ccc}
0 & l_{3} & -l_{2} \\
-l_{3} & 0 & l_{1} \\
l_{2} & -l_{1} & 0
\end{array}\right), \quad M(s)=\left(\begin{array}{ccc}
0 & m_{3} & -m_{2} \\
-m_{3} & 0 & m_{1} \\
m_{2} & -m_{1} & 0
\end{array}\right)
$$

The equation $f(s)=0$ is equivalent to the algebraic equation of fourth degree $F(s)=\prod_{j=1}^{4}\left(s-s_{j}\right)=0$, where

$$
F(s)=\left[\left(h_{1} s^{2}-h_{2} s+h_{3}\right)^{2}-4 h_{0}^{2} G(s)\right] /\left(h_{1}^{2}-4 h_{0}^{2}\right) .
$$

This equation has four roots $s_{1}, s_{2}, s_{3}$, and $s_{4}$ that, in general, are complex ones. To them correspond four complex vectors

$$
\mathbf{l}^{(p)}=\mathbf{l}\left(s_{p}\right) / \sqrt{F^{\prime}\left(s_{p}\right)}, \quad p=1,2,3,4,
$$

(here $F^{\prime}(s)$ is the derivative of $F(s)$ ) but only two of them, for example $\mathbf{l}^{(1)}$ and $\mathbf{l}^{(2)}$, are linearly independent, and

$$
\left(\mathbf{l}^{(p)}\right)^{2}=\sum_{k=1}^{3}\left(l_{k}^{(p)}\right)^{2}=0, p=1,2,3,4 ; \sum_{p=1}^{4}\left(l_{k}^{(p)}\right)^{2}=0, k=1,2,3 .
$$

Let us introduce also the vectors $\xi$ and $\eta$ by the formulae ${ }^{7}$

$$
\xi_{j}=l_{j}^{(1)}+i l_{j}^{(2)}, \quad \eta_{j}=l_{j}^{(1)}-i l_{j}^{(2)} .
$$

Using (12) and (22) we may express $m_{j}$ and $n_{j}$ in terms of $\xi_{j}$ and $\eta_{j}$

$$
m_{j}=\alpha_{j} \xi_{j}+\beta_{j} \eta_{j}, \quad n_{j}=\gamma_{j} \xi_{j}+\delta_{j} \eta_{j}
$$

where

$$
\begin{aligned}
& \alpha_{j}=\frac{\sqrt{s_{k l}^{(2)} / F^{\prime}\left(s_{2}\right)}-i \sqrt{s_{k l}^{(1)} / F^{\prime}\left(s_{1}\right)}}{\Delta_{j}^{(3)}}, \quad \beta_{j}=\frac{\sqrt{s_{k l}^{(2)} / F^{\prime}\left(s_{2}\right)}+i \sqrt{s_{k l}^{(1)} / F^{\prime}\left(s_{1}\right)}}{\Delta_{j}^{(3)}}, \\
& \gamma_{j}=\frac{\sqrt{s_{j 4}^{(2)} / F^{\prime}\left(s_{2}\right)}-i \sqrt{s_{j 4}^{(1)} / F^{\prime}\left(s_{1}\right)}}{\Delta_{j}^{(3)}}, \quad \delta_{j}=\frac{\sqrt{s_{j 4}^{(2)} / F^{\prime}\left(s_{2}\right)}+i \sqrt{s_{j 4}^{(1)} / F^{\prime}\left(s_{1}\right)}}{\Delta_{j}^{(3)}},
\end{aligned}
$$

\footnotetext{
7 As it was noted by Yu. N.Fedorov, there is relation of these vectors to the problem of geodesics on two-dimensional ellipsoid with half-axes $\sqrt{d}_{j}, \mathrm{j}=1,2,3$. Namely, $\xi$ may be considered as a tangent vector to geodesics and $i \eta$ as a normal vector to this geodesics.
} 


$$
\Delta_{j}^{(p)}=\frac{\sqrt{s_{j 4}^{(q)} s_{k l}^{(r)}-s_{j 4}^{(r)} s_{k l}^{(q)}}}{\sqrt{F^{\prime}\left(s_{q}\right) F^{\prime}\left(s_{r}\right)}} .
$$

Here $(j, k, l)$ and $(p, q, r)$ are cyclic permutations of $(1,2,3)$.

Now it is easy to check that equations (7) take the form of three Kötter's quadrics (11), where

$$
\sqrt{d_{j}}=\frac{\Delta_{j}^{(1)}-i \Delta_{j}^{(2)}}{\Delta_{j}^{(3)}}, \quad \frac{1}{\sqrt{d_{j}}}=-\frac{\Delta_{j}^{(1)}+i \Delta_{j}^{(2)}}{\Delta_{j}^{(3)}} .
$$

B. Following [Koe 1892], let us show that the manifold defined by equations (11) may be "uniformized" by means of the Weierstrass Wurzelfunctionen related to the hyper-elliptic curve (8) that are defined as

$$
\begin{aligned}
P_{j}\left(z_{1}, z_{2}\right) & =\sqrt{\left(z_{1}-d_{j}\right)\left(z_{2}-d_{j}\right)}, \quad j, k=0,1,2,3,4 \\
P_{j k}\left(z_{1}, z_{2}\right) & =\frac{P_{j} P_{k}}{\left(z_{1}-z_{2}\right)}\left(\frac{\sqrt{R\left(z_{1}\right)}}{\left(z_{1}-d_{j}\right)\left(z_{1}-d_{k}\right)}-\frac{\sqrt{R\left(z_{2}\right)}}{\left(z_{2}-d_{j}\right)\left(z_{2}-d_{k}\right)}\right)
\end{aligned}
$$

These sixteen functions $P_{j}\left(z_{1}, z_{2}\right)$ and $P_{j k}\left(z_{1}, z_{2}\right)$ satisfy a lot of identities. All of them may be obtained from definitions (27) and (28) (for details, see [We 1878], [Koe 1892], and [Ca 1893]). ${ }^{8}$ Here we give only few of them which are useful for us:

$$
\begin{gathered}
\sum_{j=1}^{3} c_{j}\left(\frac{P_{k l}^{2}}{\left(s-d_{k}\right)\left(s-d_{l}\right)}+\frac{P_{j 4}^{2}}{\left(s-d_{j}\right)\left(s-d_{4}\right)}\right)=\frac{s}{\prod_{j=1}^{4}\left(s-d_{j}\right)}, \\
\sum_{j=1}^{3} \tilde{c}_{j} P_{j 4}^{2}=d_{4}, \quad \sum_{j=1}^{3} d_{j} \tilde{c}_{j} P_{k l}^{2}=P_{0}^{2}, \\
\sum_{j=1}^{3} c_{j} P_{j 4} P_{k l}=0, \quad \sum_{j=1}^{3} \tilde{c}_{j} P_{j 4} P_{k l}=-P_{0}, \\
\sum_{j=1}^{3} c_{j}\left(d_{j}^{-1} P_{j 4}^{2}+d_{j} P_{k l}^{2}\right)=0,
\end{gathered}
$$

\footnotetext{
${ }^{8}$ See also modern survey [BEL 1997].
} 
where

$$
\tilde{c}_{j}=\frac{1}{\left(d_{j}-d_{k}\right)\left(d_{j}-d_{l}\right)}, \quad c_{j}=\frac{d_{j}-d_{4}}{\left(d_{j}-d_{k}\right)\left(d_{j}-d_{l}\right)} .
$$

It is known (see [We 1878]) that $P_{j}\left(z_{1}, z_{2}\right)$ and $P_{j k}\left(z_{1}, z_{2}\right)$ up to the factors are the ratio of the theta functions with half-integer theta characteristics ${ }^{9}$

$$
\begin{array}{cc}
P_{j}\left(z_{1}, z_{2}\right)=f_{j}\left(u_{1}, u_{2}\right)=\frac{\theta_{j}\left(u_{1}, u_{2}\right)}{\theta\left(u_{1}, u_{2}\right)}, & P_{k l}\left(z_{1}, z_{2}\right)=f_{k l}\left(u_{1}, u_{2}\right)=\frac{\theta_{k l}\left(u_{1}, u_{2}\right)}{\theta\left(u_{1}, u_{2}\right)}, \\
\theta_{23}\left(u_{1}, u_{2}\right)=\theta\left[\begin{array}{ll}
0 & 0 \\
1 & 1
\end{array}\right]\left(u_{1}, u_{2}\right), & \theta_{31}\left(u_{1}, u_{2}\right)=\theta\left[\begin{array}{ll}
1 & 0 \\
1 & 1
\end{array}\right]\left(u_{1}, u_{2}\right), \\
\theta_{12}\left(u_{1}, u_{2}\right)=\theta\left[\begin{array}{ll}
1 & 0 \\
0 & 0
\end{array}\right]\left(u_{1}, u_{2}\right), & \theta_{14}\left(u_{1}, u_{2}\right)=\theta\left[\begin{array}{ll}
1 & 1 \\
1 & 1
\end{array}\right]\left(u_{1}, u_{2}\right), \\
\theta_{24}\left(u_{1}, u_{2}\right)=\theta\left[\begin{array}{ll}
0 & 1 \\
1 & 1
\end{array}\right]\left(u_{1}, u_{2}\right), & \theta_{34}\left(u_{1}, u_{2}\right)=\theta\left[\begin{array}{ll}
0 & 1 \\
0 & 0
\end{array}\right]\left(u_{1}, u_{2}\right), \\
\theta_{0}\left(u_{1}, u_{2}\right)=\theta\left[\begin{array}{ll}
1 & 1 \\
0 & 0
\end{array}\right]\left(u_{1}, u_{2}\right), & \theta\left(u_{1}, u_{2}\right)=\theta\left[\begin{array}{ll}
0 & 0 \\
0 & 0
\end{array}\right]\left(u_{1}, u_{2}\right) .
\end{array}
$$

Here

$$
\theta\left(u_{1}, u_{2}\right)=\sum_{n_{1}, n_{2}} \exp \left\{i \pi\left(n_{1}\left(2 u_{1}+n_{1} \tau_{11}+n_{2} \tau_{12}\right)+n_{2}\left(2 u_{2}+n_{1} \tau_{21}+n_{2} \tau_{22}\right)\right)\right\},
$$

and $\tau_{j k}$ are elements of period matrix.

The comparison (11) with (29)-(33) shows that

$$
\xi_{j}=\sqrt{c_{j}} g P_{k l}, \quad \eta_{j}=\sqrt{c_{j}} g P_{j 4},
$$

where $g$ is an unknown function.

C. The rest part of the proof is the uniformization of equation (6).

Let us substitute expressions (23) for $m_{j}$ and $n_{j}$ into equation (6). Then by using of (24) and (25) we transform it to the form

$$
H_{0}=\sum_{j=1}^{3}\left(A_{j}\left(\xi_{j}^{2}-\eta_{j}^{2}\right)+B_{j} \xi_{j} \eta_{j}\right)=h_{0},
$$

\footnotetext{
${ }^{9}$ We give here just one series of such expressions. Relative other series, see [Koe 1892].
} 
where

$$
A_{j}=\alpha+\beta d_{j}+\gamma d_{j}{ }^{-1}, \quad B_{j}=\delta\left(d_{j}+d_{j}^{-1}\right) .
$$

Here $\alpha, \beta, \gamma$, and $\delta$ are algebraic functions of $h_{0}, h_{1}, h_{2}, h_{3}, a_{j}$, and $b_{j}$.

This sum may be calculated by using of (24), (29)-(33). The result is

$$
H_{0}=\frac{\left(1-\varepsilon P_{0}\right)^{2}}{4 \varepsilon d_{4}} g^{2}=h_{0},
$$

where

$$
\varepsilon=\frac{\sqrt{d_{4}}\left(\sqrt{\left(s_{3}-s_{1}\right)\left(s_{2}-s_{4}\right)}-\sqrt{\left(s_{2}-s_{3}\right)\left(s_{1}-s_{4}\right)}\right)}{\sqrt{\left(s_{1}-s_{2}\right)\left(s_{3}-s_{4}\right)}} .
$$

From this we obtain

$$
g=\left(1-\varepsilon f_{0}\right)^{-1}, \quad \xi_{j}=g \sqrt{c_{j}} f_{k l}, \quad \eta_{j}=g \sqrt{c_{j}} f_{j 4} .
$$

The fact of linear dependence of arguments $u_{1}$ and $u_{2}$ on time $t$ follows as from the algebraic geometrical approach [AM 1982] as from the old Kötter approach [Koe 1892].

This completes the proof of Theorem.

Acknowledgements. The main part of this paper was completed during my visit to Max-Planck Institute for Mathematics, Bonn. I am grateful to the stuff of this Institute and also to the Department of Theoretical Physics of Zaragoza University for hospitality.

\section{References}

[AM 1982] Adler M. and van Moerbeke P., The algebraic integrability of geodesic flow on $S O(4)$, Inv. Math. 67, 297-326; with appendix by D. Mumford, pp. 326 - 331

[AM 1988] Adler M. and van Moerbeke P., The Kowalewski and HénonHeiles motions as Manakov geodesic flows on $S O(4)$ - a two-dimensional family of Lax pairs, CMP 113, 659-700

[BEL 1997] Bukhstaber V.M., Enolskii V.Z. and Leikin D.V. Hyperelliptic Kleinian functions and applications, Solitons, geometry, and topology: on crossroad, 1-33, Amer. Math. Soc. Transl. Ser 2, 179, Amer. Math. Soc., Providence, RI 
[Bo 1986] Bobenko A.I., Euler equations on the Lie algebras e(3) and so(4). Isomorphism of integrable cases, Funct. Anal. Appl. 20, 53-56

[Ca 1893] Caspary F., Sur une nouvelle manière d'établir les relations algébriques qui ont lieu entre les fonctions hyperelliptiques de première espèce, Ann. Sci. Ec. Norm. Sup., 3 Ser., 10, 253-294

[Ca 1846] Cayley A., Sur quelques propriétés des détérminants gauches, J. Reine Angew. Math. 32, 119-123

[Cl 1871] Clebsch A., Über die Bewegung eines Körpers in einer Flüssigkeit, Math. Ann. 3, 238-261

[Fe 2000] Fedorov Yu.N., Integrable systems, Poisson pencils, and hyperelliptic Lax pairs, Reg. Chaot. Dyn. 5, No.2, 171-180

[Fr 1874] Frahm F., Über gewisse Differentialgleichungen, Math. Ann. 8, 3544

[Ha 1983] Haine L., Geodesic flow on SO(4) and abelian surfaces, Math. Ann. 263, No.4, 435-472

[Koe 1892] Kötter F., Über die Bewegung eines festen Körpers in einer Flüssigkeit, I, II, J. Reine Angew. Math. 109, 51-81, 89-111

[Ma 1977] Manakov S.V., Note on the integration of Euler's equations of the dynamics of an n-dimensional rigid body, Funct. Anal. Appl. 10, 328-329

[Mo 1980] Moser Ju., Geometry of quadrics and spectral theory, in: Chern Symposium 1979, 147-188

[Sch 1891] Schottky F., Über das analytische Problem der Rotation eines starren Körpers im Raume von vier Dimensionen, Sitzungber. König. Preuss. Akad. Wiss. zu Berlin, 227-232

[We 1878] Weber H., Anwendung der Thetafunctionen zweiter Veränderlicher auf die Theorie der Bewegung eines festen Körpers in einer Flüssigkeit, Math. Ann. 14, 173-206 